\title{
Spatial Diversity Effect on the Experimental Gain of Capacity of the Outdoor Mobile Radio Channel in the $700 \mathrm{MHz}$ Band
}

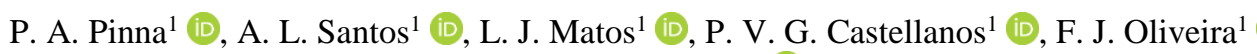 \\ and G. L. Siqueira ${ }^{2}$ iD \\ ${ }^{1}$ Graduate Program of Telecommunications Engineering, Fluminense Federal University, Niterói, Brazil, \\ paula.a.pinna@gmail.com, andrea.ouro@hotmail.com,lenijm@id.uff.br,pedroc@id.uff.br, \\ fernjos86@gmail.com \\ ${ }^{2}$ Pontifical Catholic University of Rio de Janeiro CETUC/PUC-Rio, Rio de Janeiro, Brazil \\ glaucio.siqueira@puc-rio.br
}

\begin{abstract}
From measurements performed in the $700 \mathrm{MHz}$ band, this paper analyzes the effect of spatial diversity on the channel capacity through the propagation of an OFDM mobile radio signal and compares with the results of a single branch providing what improvement of the capacity is attained with the SIMO system.
\end{abstract}

Index Terms - channel capacity; power-delay profile; signal dispersion; spatial diversity.

\section{INTRODUCTION}

With the released band of open TV in Brazil, the cellular systems could operate in $700 \mathrm{MHz}$ band, which is interesting for the cellular providers mainly because of the range of the signal, providing economy for them due to the smaller number of base stations for covering some area.

The fourth generation (4G) system is already operating in many Brazilian cities and tests are been performed in several of them. It works with the LTE (Long Term Evolution) technology, using OFDM (Orthogonal Frequency Division Multiplexing) signal.

For better understanding the radio mobile channel behavior in $700 \mathrm{MHz}$ band authors have published different papers. Matolak deals with experimental outdoor-to-indoor channel dispersion in 700 and $4900 \mathrm{MHz}$ [1]; Gentile reports models developed for peer-to-peer wireless channels for an urban environment in the $700 \mathrm{MHz}$ and $4.9 \mathrm{GHz}$ bands, both allocated for public safety and "emergency responder" applications [2]. Moura applies artificial neural network for predicting the signal coverage in outdoor-to-indoor in $768 \mathrm{MHz}$, concluding about the improvement of prediction with a simple MLP (Multilayer Perceptron) network [3]. Cueto deals with simulations in 700 and $2600 \mathrm{MHz}$ and compares coverage and capacity of LTE-Advanced Networks in $700 \mathrm{MHz}$ and 2.6 $\mathrm{GHz}$ [4]. Several references deal with the reception of OFDM signal in spatial diversity. Among them: blind estimation techniques that exploit the receive antenna diversity [5]; effects of diversity in the receiving antenna in a multipath fading channel [6]; analysis of the macro-diversity in LTEAdvanced [7]. However, those studies were with simulations. In this context, this paper provides experimental results for the capacity obtained in $700 \mathrm{MHz}$ band using the spatial diversity in a SIMO (Single-Input Multiple-Output) system in an outdoor mobile radio channel. It is fundamental 
performing measurements in order to corroborate the simulations. Then, the purpose is to determine the channel capacity in a SIMO system from the experimental gain of spatial diversity on the reception of an OFDM mobile radio signal. To accomplish this, the paper follows with Section II, which describes the theoretical aspects necessary to calculate the channel capacity. Section III describes the transmission and reception systems and the measurement environment whereas Section IV provides the results. Section V presents the conclusions.

\section{THEORETICAL ASPECTS}

\section{A. Mobile Radio Channel Transfer Function}

The randomness of the mobile radio channel makes stochastic the transfer function $h$, that defines the behavior of the propagation channel [8]. Based on the measurements performed, this function is experimentally determined for the $760 \pm 10 \mathrm{MHz}$ band in both diversity branches: transmitter-receiver 1 (TX-RX1) and transmitter-receiver 2 (TX-RX2). Based on [8,9] if the received signal passes through a filter matched to the transmitted signal $(s(t))$, the transfer function of the channel is the output of this filter when an impulsive input is used. Then, the filtering process is equivalent to a correlation process $\left(R_{S}\right)$, which will result in the instantaneous transfer function $h\left(t_{i}, \tau\right)$ of the channel probed along the delays:

$$
h\left(t_{i}, \tau\right)=\sum_{i=1}^{N} C_{i} R_{S}\left(t_{i}-\tau_{i}, \tau\right)
$$

in which $\tau$ stands for delay, $C_{i}$ is the complex amplitude of the received signal $C_{i}=\operatorname{Re}\left(s^{\prime}\right)+j \operatorname{Im}\left(s^{\prime}\right)$ and $s^{\prime}$ is the quadrature signal measured at the reception by the signal analyzer. From $h(t, \tau)$, the mean delay and delay spread parameters, which quantify the temporal dispersion of the signal in the transmission channel, can be determined. For this purpose, in each $t_{i}$, instantaneous power delay profiles are calculated from [8]:

$$
P_{h}\left(t_{i}, \tau\right)=\left|h\left(t_{i}, \tau\right)\right|^{2}
$$

The average delay $(\bar{\tau})$ is calculated as the first center moment, or the average, of the instantaneous power delay profile $P_{h}(\tau)$. Due to the discrete acquisition, $P_{h}\left(\tau_{i}\right)$ is the relative power on the delay $\tau_{i}$, with $N$ representing the number of correlation peaks within a power delay profile (named PDP), which characterizes the valid multipath. Then, its definition is:

$$
\bar{\tau}=\frac{\sum_{i=1}^{N-1} \tau_{i} P_{h}\left(\tau_{i}\right)}{\sum_{i=0}^{N-1} P_{h}\left(\tau_{i}\right)}
$$

The RMS delay spread $\left(\sigma_{T}\right)$ is the standard deviation of the probability density function, which characterizes the arrival time of the multipath in the receiver [8]. This is calculated by the square root of the second center moment, variance, of the power delay profile $P_{h}(\tau)$. In discrete form, the RMS delay spread is [8]:

$$
\sigma_{T}=\sqrt{\frac{\sum_{i=1}^{N-1}\left(\tau_{i}-\bar{\tau}\right)^{2} P_{h}\left(\tau_{i}\right)}{\sum_{i=0}^{N-1} P_{h}\left(\tau_{i}\right)}}
$$




\section{B. Channel capacity}

For a memoryless system, the maximum capacity of a wideband SISO system $\left(C_{S I S O}\right)$ in a Gaussian channel is [10]:

$$
C_{\text {SISO }}=B \cdot \log _{2}\left(1+\rho|h|^{2}\right)
$$

with $\rho$ meaning the mean signal-to-noise ratio $\left(S N R=S / P_{N}\right)$ at the receiving antenna, $h$ is the complex channel gain calculated as described previously, and $B$ is the band of the signal. The same process occurs in memoryless wideband SIMO system, in which each reception channel is independent from the other. In this case, if $\rho$ is a constant mean in the environment of a Gaussian noise, the maximum channel capacity, in bps, is calculated from:

$$
C_{\text {SIMO }}=B \cdot \log _{2}\left(1+\rho \sum_{i=1}^{N}\left|h_{i}\right|^{2}\right)
$$

in which $h_{i}$ is the channel gain related to each branch of diversity, calculated from (1), therefore, $\left|h_{i}\right|^{2}$ means the absolute value of the power delay profile, calculated from (2), and $N$ is the number of receiving antennas. In a random time variant channel, the maximum capacity due to the diversity in a SIMO receiver $\left(C_{S I M O}\right)$ is [10]:

$$
C_{\text {SIMO }}=B \cdot \log _{2}\left\{\operatorname{det}\left[1+\rho H H^{H}\right]\right\}
$$

with $H$ meaning the $N X I$ channel gain, $H^{H}$ is the transposed matrix of $H$, and $N$ is the number of receiving antennas. If two diversity branches are used:

$$
\left.C_{\text {SIMO }}=B \cdot \log _{2}\left\{1+\rho\left|H_{11}\right|^{2}+\rho\left|H_{12}\right|^{2}\right]\right\}
$$

in which $H_{11}$ and $H_{12}$ represent the channel gains on the diversity branches TX-RX1 and TX-RX2, respectively, i.e., $H_{11}=h_{1}\left(t_{i}, \tau\right)$ and $H_{12}=h_{2}\left(t_{i}, \tau\right)$. It is noteworthy that for different points, with different distances from the receiver to the transmitter, the value of the SNR will be different and the normalized gain of the channel will be different in each situation too.

\section{SETUP SPECIFICATIONS AND MEASUREMENT ENVIRONMENT}

The measurements were carried out in the neighborhood of Higienópolis, suburb of the city of Rio de Janeiro, Brazil, in a suburban environment with high vehicular traffic and many constructions, following the route shown in Fig.1. The transmitting station was at the top of a fourteen floorsbuilding located at Dom Helder Câmara Street, 3152, in the neighborhood of Del Castilho. The receiving station was in a van equipped with the full OFDM signal capture system transmitted on the carrier frequency of $760 \mathrm{MHz}$ in a band of $20 \mathrm{MHz}$.

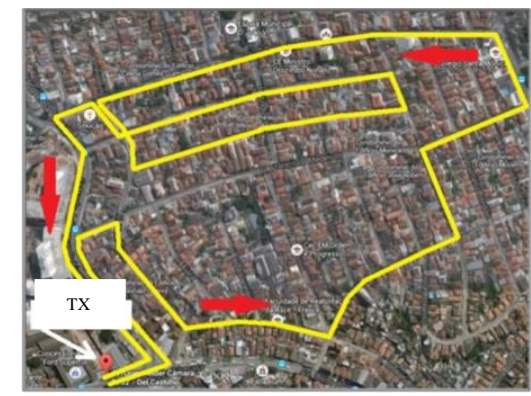

Fig. 1. Route sounded in the neighborhood of Higienópolis.

Brazilian Microwave and Optoelectronics Society-SBMO received 28 Nov 2019; for review 5 Dec 2019; accepted 5 March 2020 Brazilian Society of Electromagnetism-SBMag 


\section{A. Measurement Setup Description}

The systems specifications used in the channel sounding are in Table I and the block diagram is on Fig. 2.

TABLE I. SPECIFICATIONS OF THE SYSTEM (TX-TRANSMITTER/RX-RECEIVER)

\begin{tabular}{lll}
\hline \multicolumn{1}{c}{ Device } & Specification \\
\hline \multirow{4}{*}{ VX } & Vector signal generator MG3700A, Anritsu & $250 \mathrm{kHz}-6 \mathrm{GHz}$ \\
2 cables RG 213 & $1 \mathrm{~m} / 0.2 \mathrm{~dB}$ loss; $6 \mathrm{~m} / 1.33 \mathrm{~dB}$ loss \\
Power Amplifier & ZHL-16W-43+ \\
Digital Power Source & PS-5000, ICEL \\
Sectorial Antenna APX75-866512-CTO, RFS & $65^{\circ}$ beam aperture/698-896 MHz/ 14 dBi \\
\hline \multirow{4}{*}{ Vector signal analyzer MS-2692A, Anritsu } & $50 \mathrm{~Hz}-26.5 \mathrm{GHz}$ \\
Antenna CE-150727, CELTA & $2 \mathrm{dBi}$ in 760 MHz \\
Low Noise Amplifier & $2 \mathrm{LNA}$ ZX60-33LN-S+/ $19.5 \mathrm{~dB}$ \\
RX & GarmimMAP 64S \\
2 Laptops & Dell Inspiron \\
2 Cables RG 213 & $1 \mathrm{~m} / 0.2 \mathrm{~dB}$ loss; $6 \mathrm{~m} / 1.33 \mathrm{~dB}$ loss \\
2 Network cables & \\
\hline
\end{tabular}
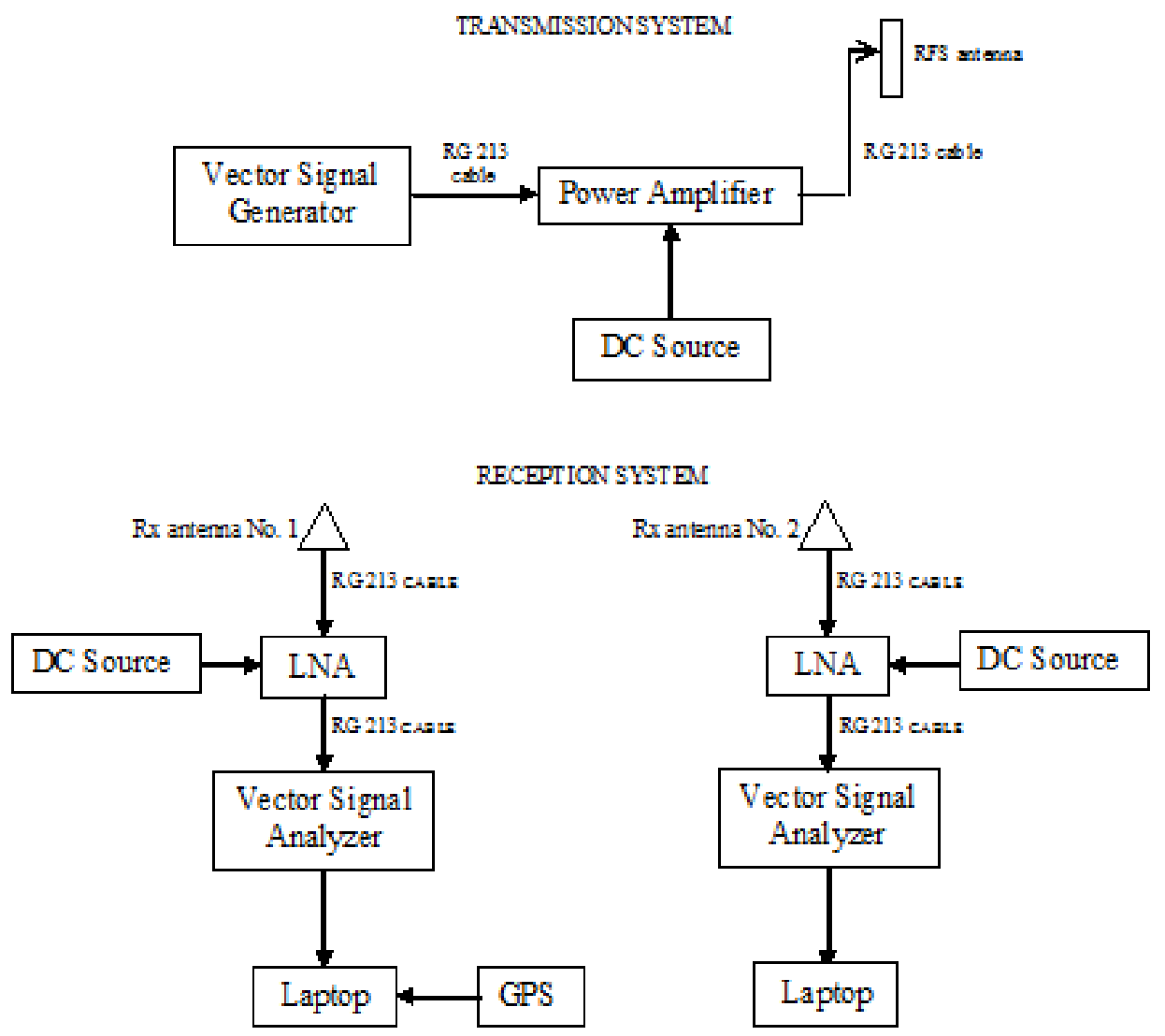

Fig. 2. Transmission and reception system.

A $20 \mathrm{MHz}$ bandwidth OFDM signal generated in $\mathrm{Matlab}^{\circledR}$ software and transferred to the signal vector generator by I and Q components, is composed of 1024 subcarriers, with 128 samples of cyclic 
prefix. These values were converted to the time domain. The cyclic prefix has been inserted and the components (real and imaginary) have been separated to form the in-phase and quadrature components of the OFDM signal, which is carried by the network cable to the signal generator.

The high power amplifier (PA), with a gain of approximately $36 \mathrm{~dB}$ for the $760 \mathrm{MHz}$ frequency, connected the output of the signal generator to the transmitting antenna in order to provide a greater coverage of the signal. The connection was via the RG 213-50 ohms cable with $6 \mathrm{~m}$ long, and -11 $\mathrm{dBm}$ was the output of the vector signal generator, considering the saturation curve of the PA used. Thus, at the input of the $14 \mathrm{dBi}$ antenna, the signal level was equal to $22.67 \mathrm{dBm}$, totaling $36.67 \mathrm{dBm}$ at the antenna output. This antenna was in a mast of $3 \mathrm{~m}$ height, at the top of a building with 45 meters in height, totaling 48 meters, located at latitude S $22.878174^{\circ}$ and longitude W $43.265450^{\circ}$.

For the reception, the antennas were installed on the roof of the vehicle at a chosen distance of 53 $\mathrm{cm}$ from each other, equivalent to $1.35 \lambda$, which guarantees a negligible correlation coefficient between the signals received by both, providing effective diversity [8]. The RG213 cables conducted the signals to the LNA inputs of each receiver system. Each LNA was connected to a MS-2692A signal analyzer, used for capturing the data transferred to a laptop via network cable. For the location of the measurements, it was used a GPS connected to one of the laptops through USB cable.

\section{RESULTS}

Throughout the channel sounding, samples of the two receiving antennas, 1 and 2, were collected simultaneously, with the sampling rate being 50 MSPS (Mega Samples Per Second), as suggested by the equipment manual, for a $20 \mathrm{MHz}$ signal bandwidth received. After processing the received data (I and Q), the PDPs were calculated according to (2) by using (1), and the mean delay and RMS delay spread values obtained from the calculated profiles are shown, respectively, in Fig. 3 and Fig. 4. A predominance of values below 0.04 microseconds and 0.15 microseconds, respectively, occurs for the average delays of antennas 1 and 2 whereas the delay spread is below 0.3 and 0.08 microseconds, respectively, for the antennas 1 and 2 .

At the beginning of the route, both the left and the right antenna presented higher values of delay spread. This behavior is due to the proximity of the receiving antennas to the transmitter, where the signal is stronger, therefore there is greater contribution of multipath in reception. Their variation in the whole route was in the range of 0.0069 to 1.4093 microseconds for the antenna 1 and fractions of nanoseconds to 0.410 microseconds for the antenna 2. Then, for the range of $760 \mathrm{MHz}$, the temporal dispersion results are closer to a suburban/less dense urban region [12]. 


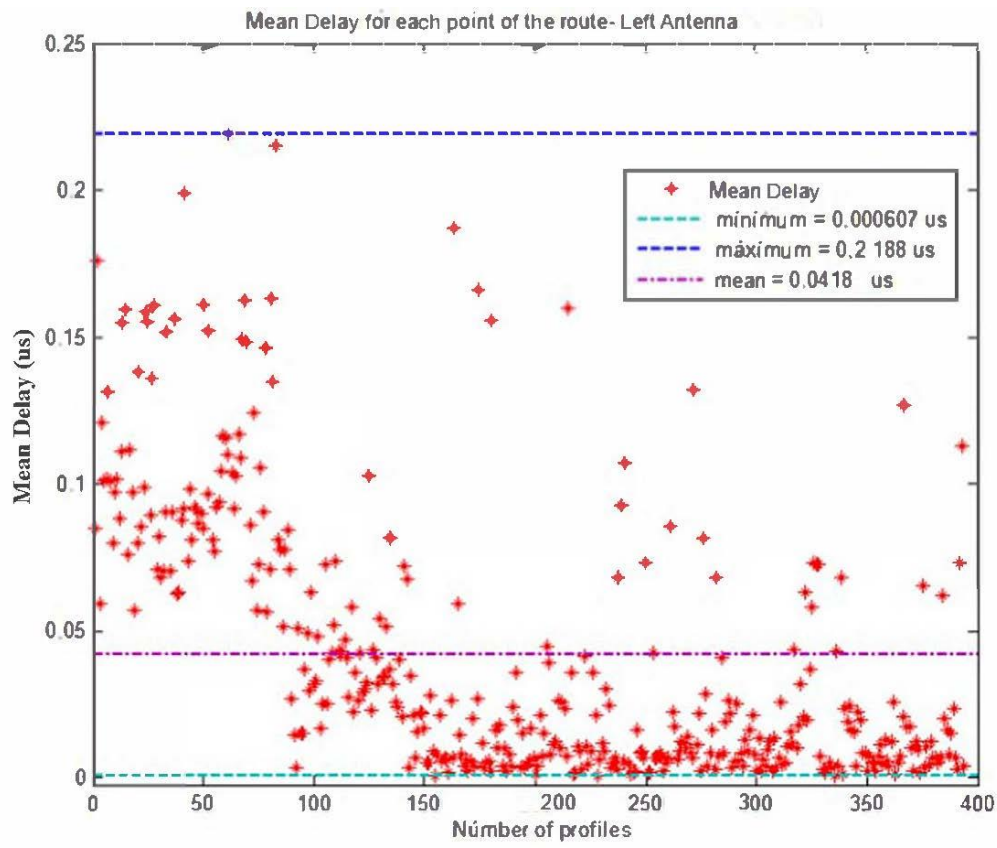

(a)

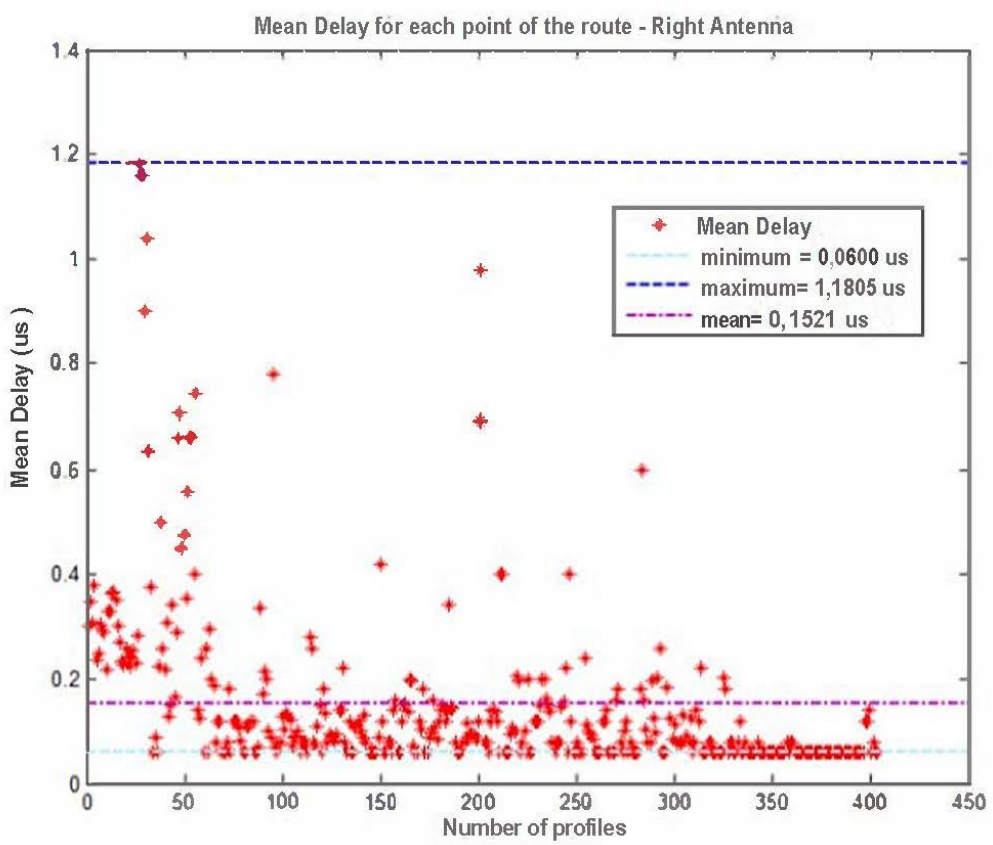

(b)

Fig. 3. Mean delay versus number of PDPs; (a) Antenna 1 and (b) Antenna 2. 


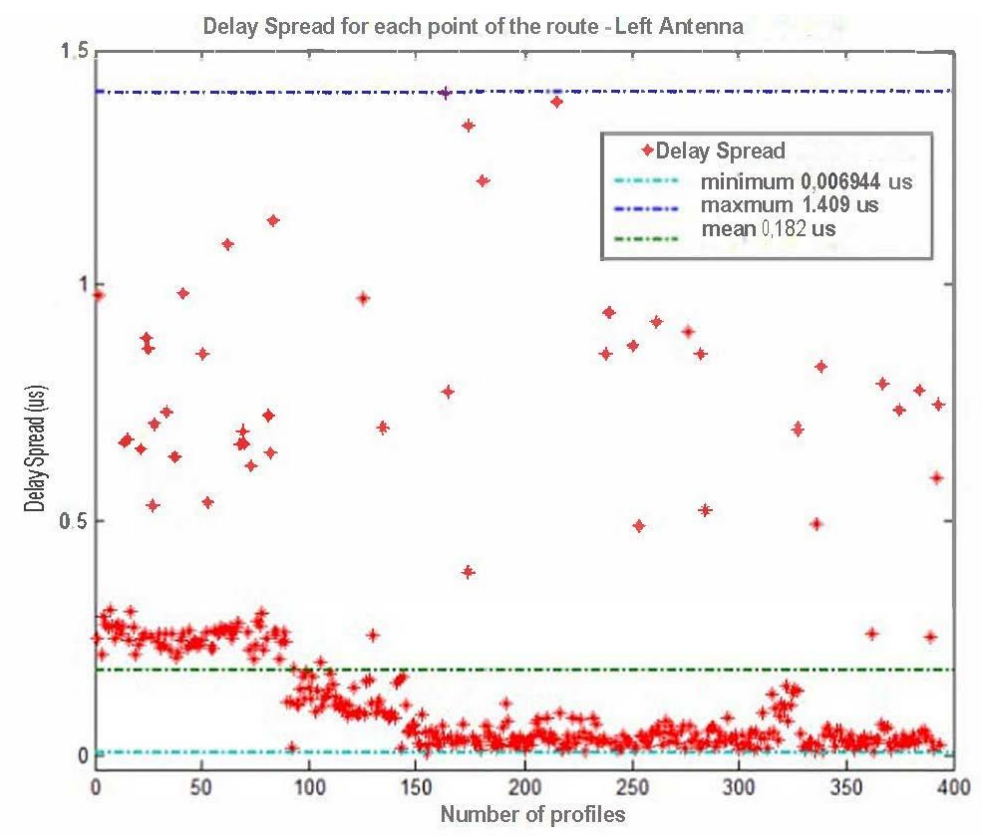

(a)

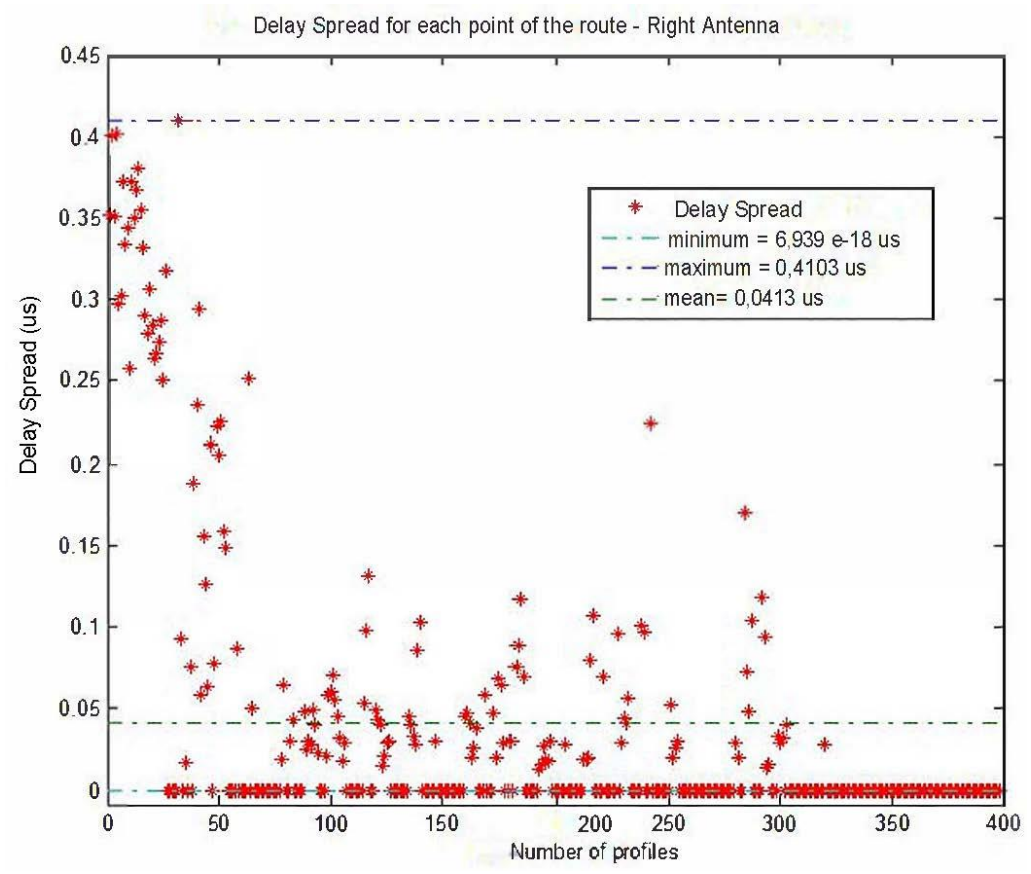

(b)

Fig. 4. Delay spread versus number of PDPs; (a) Antenna 1 and (b) Antenna 2.

In order to obtain the mean signal-to-noise ratio $\rho$ used in (8), the mean signal power was calculated for each received OFDM signal (I and Q) and the mean noise power $\left(P_{N}\right)$ in the symbol band is calculated fusing equations 6-18 from [13]:

$$
P_{N}=10 \log _{10}\left[B_{S} \cdot \frac{n \sum_{i=1}^{n} 10^{P i / n}}{1.065 . R B W}\right]
$$


and $R B W(3 \mathrm{kHz})$ is the band of the resolution filter setting in the spectrum analyzer used to measure the noise whose multiplication by the factor 1.065 represents the noise bandwidth of the IF filter (digital Gaussian filter) of the spectrum analyzer; $B_{S}$ is the band of the transmitted signal $(20 \mathrm{MHz}), n$ is the number of the noise samples (366) inside the band of $20 \mathrm{MHz}$ and $P_{i}$ is the power of each noise sample. In our measurements was used a $30 \mathrm{MHz}$ span in a 551 points spectrum analyzer. The value of $n$ depends on the number of trace samples of the spectrum analyzer. It is worth mentioning that $P_{i}$ samples were acquired along the routes without turning on the transmitter, only capturing noise samples, and were saved for post processing. The measured noise of the channel was $-141 \mathrm{dBm} / \mathrm{Hz}$. In the $700 \mathrm{MHz}$ band, the ambient noise floor was limited by the noise floor of the measurement system. Gaussian statistics fitted very well to the amplitude samples of the noise, as illustrated in Fig. 5.

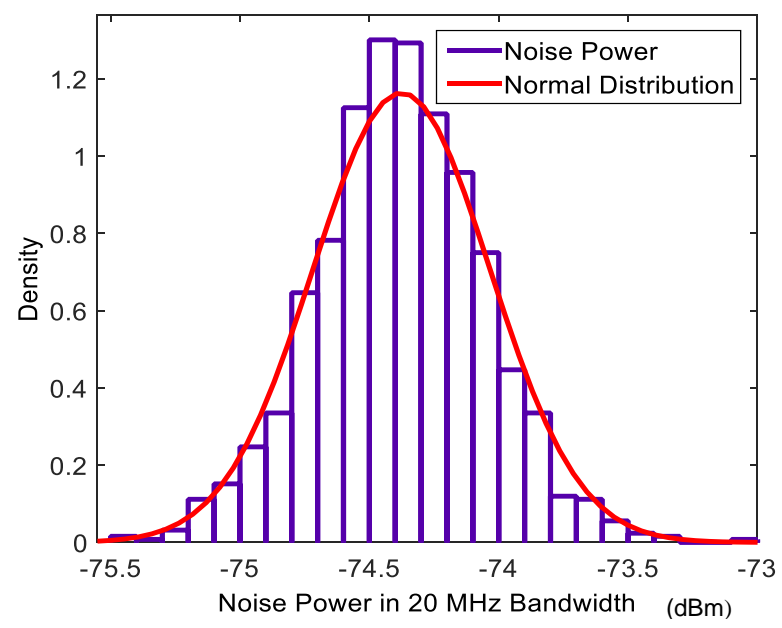

Fig. 5. Probability density function of the noise power of the channel.

As the noise presents normal statistics, (5) and (8) are used, respectively, for calculating SISO and SIMO system capacity. Therefore, firstly the capacity $(C / B)$ of each receiving branch is calculated from (5) and the results are in Fig. 6. It is worth remembering that the channel function is timevariant, thus leading to different channel capacity values, which are decreasing with the increasing distance to the transmitter, as shown in that figure. This drop is natural because the level of the received signal decreases with the distance. Besides $700 \mathrm{~m}$, the individual capacity was very small and it has been neglected. 


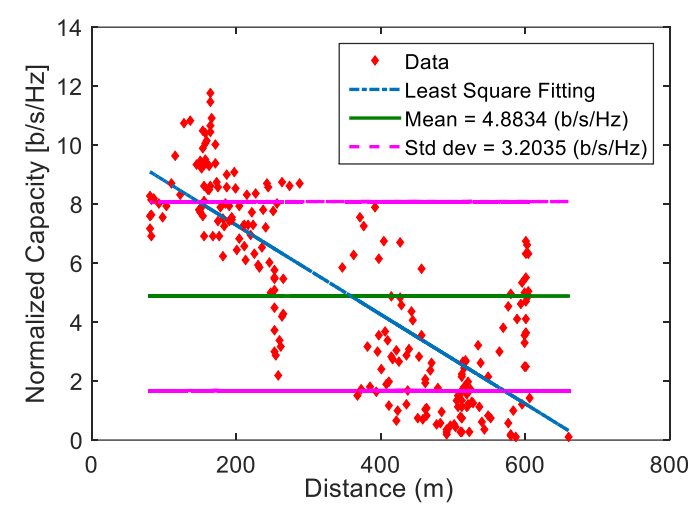

(a)

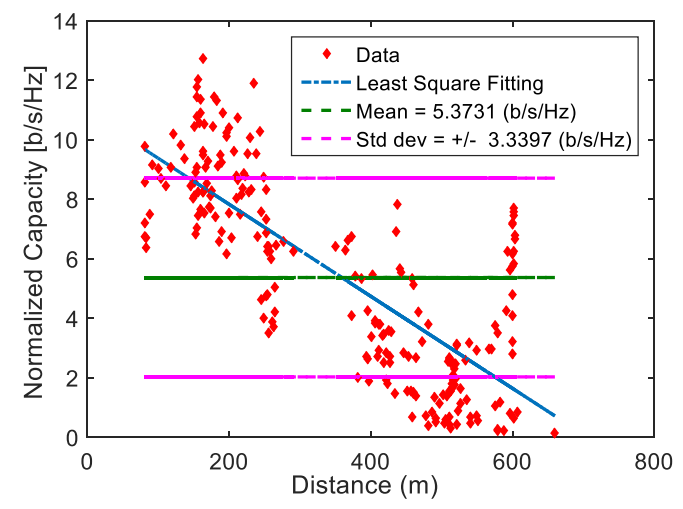

(b)

Fig. 6. Individual capacity for each antenna; (a) Antenna 1 and (b) Antenna 2.

Complementing the results, Table II provides the variation of the capacity, normalized by the 20 MHz band, related to both antennas.

The maximum capacity values obtained for both antennas and also the behavior of decreasing with the distance were very close. Another similar behavior observed in both antennas was a peak at points close to $600 \mathrm{~m}$ distance from the transmitter. This behavior occurs because the presence of direct sight in this region.

TABLE II. NORMALIZED CAPACITY BY THE 20 MHz BAND

\begin{tabular}{cccc}
\hline Antenna & Normalized capacity $[\mathbf{b} / \mathbf{s} / \mathbf{H z}]$ & Mean $[\mathbf{b} / \mathbf{s} / \mathbf{H z}]$ & Standard deviation $[\mathbf{b} / \mathbf{s} / \mathbf{H z}]$ \\
\hline 1 & $0.092<\mathrm{C} / \mathrm{B}<11.74$ & 4.88 & 3.20 \\
2 & $0.129<\mathrm{C} / \mathrm{B}<12.75$ & 5.37 & 3.34 \\
\hline
\end{tabular}

Fig. 7 depicts the distributions that best fit the normalized capacity values for each antenna. For the antenna 1, the cumulative distributions that best fit to the data were the exponential, for the smaller capacity values, and the normal, for the larger values. For antenna 2, they were normal and Nakagami distributions. The last, with low-value for the parameter $m$ (distribution parameter of Nakagami probability density function) [8] best fitted to the lowest normalized capacity values up to approximately $5 \mathrm{~b} / \mathrm{s} / \mathrm{Hz}$. This distribution, with $m$ values in the range $0.5 \leq m<1.0$ indicates low SNR, worse than Rayleigh, that is, the power of the received signal is due to weaker multipath, without a dominant path, which explains the smaller values of the normalized capacity. The normal distribution was best adjusted for normalized capacity values greater than $5 \mathrm{~b} / \mathrm{s} / \mathrm{Hz}$. Recalling that the normal distribution is a Rice distribution with factor $K \gg 1$ [8], the environment is characterized by the presence of a dominant path, which suggests that there is sight to the transmitter.

For the calculation of the joint capacity, the profiles of the left (1) and right antennas (2), synchronized, corresponding to the points of the same coordinate, were accounted. After synchronization, the SIMO system capacity according to (8), or the "joint capacity" resulted in: 


$$
0.236<\mathrm{C}_{\text {joint }} / \mathrm{B}<13.019
$$

with the mean equal to $6.273 \mathrm{~b} / \mathrm{s} / \mathrm{Hz}$ and standard deviation equal to $3.420 \mathrm{~b} / \mathrm{s} / \mathrm{Hz}$. For the $20 \mathrm{MHz}$ band used, this joint capacity varied from 4.72 to $260.38 \mathrm{Mbps}$, with $125.46 \mathrm{Mbps}$ of mean.

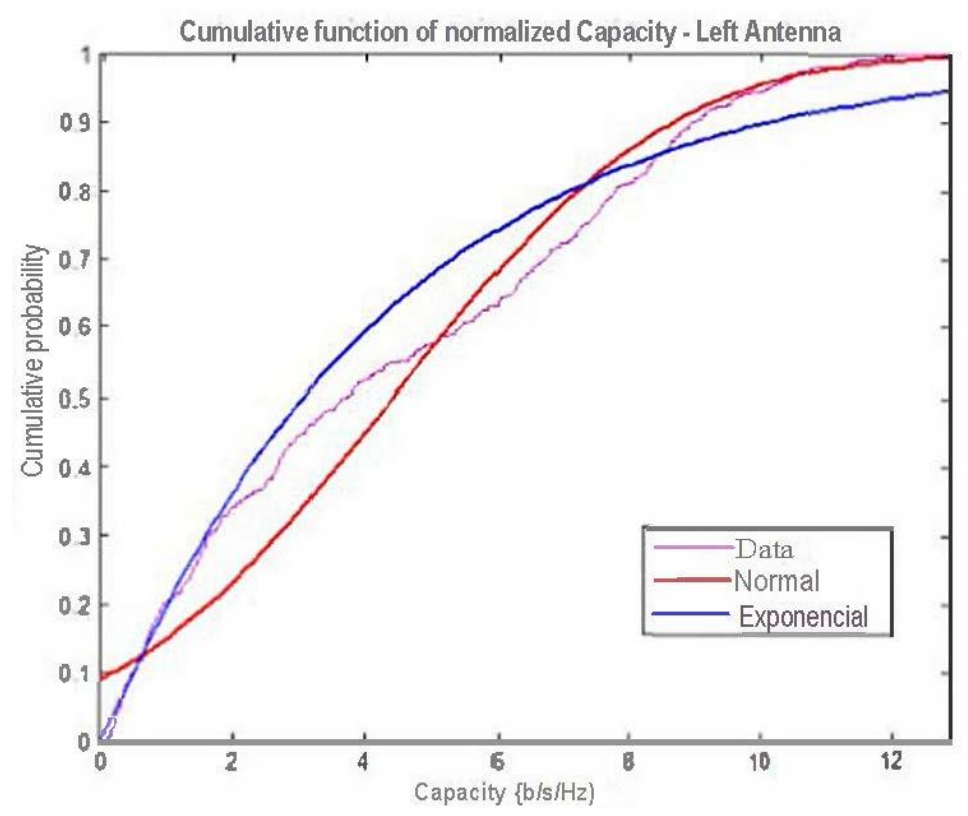

(a)

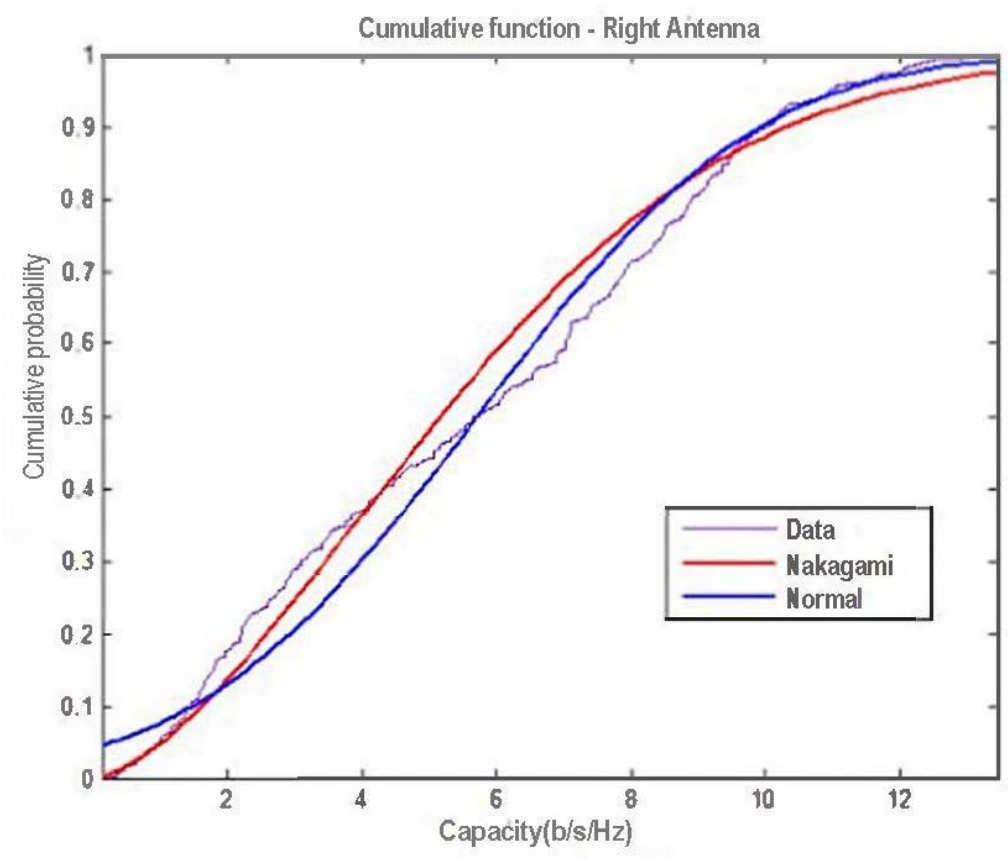

(b)

Fig. 7. Cumulative distribution function of normalized capacity; (a) Antenna 1 and (b) Antenna 2.

For observing better the capacity gain of the SIMO system, Fig. 8 provides the difference between the joint capacity and the individual capacity of each antenna, showing that the diversity effect of the 
SIMO system has produced improvement in the channel capacity, which increased from 0.05 to 6.15 $\mathrm{b} / \mathrm{s} / \mathrm{Hz}$, that is, from $1 \mathrm{Mbps}$ to $123 \mathrm{Mbps}$, depending on the distance and the channel conditions. Relatively to antenna 1, the capacity increased in the range $0.05-4.6 \mathrm{~b} / \mathrm{s} / \mathrm{Hz}$, that is, 1 to $92 \mathrm{Mbps}$. On the other hand, the capacity increased in the range $0.05-6.15 \mathrm{~b} / \mathrm{s} / \mathrm{Hz}$, corresponding to $1-123 \mathrm{Mbps}$ of improvement, when related to the antenna 2.

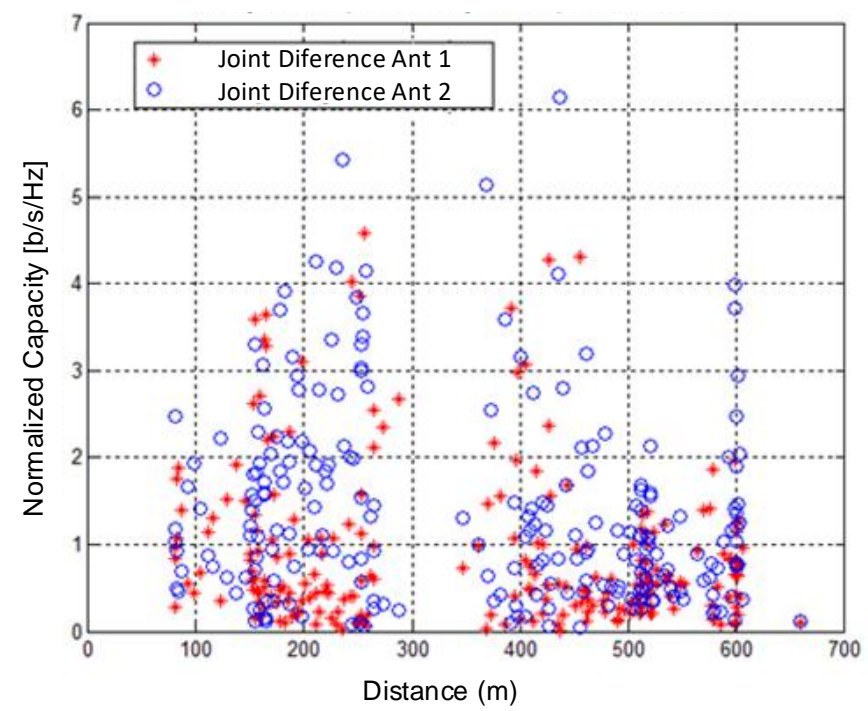

Fig. 8. Difference between joint and individual capacity.

\section{CONCLUSION}

Broadband measurements in a suburban area of Rio de Janeiro city were carried out using two independent reception branches. Throughout the routes, signal samples of the two receiving antennas, 1 and 2, were collected simultaneously, with 50 MSPS sampling rate. The transmitted signal was a 20 $\mathrm{MHz}$ OFDM. The antennas were installed on the roof of the vehicle at a chosen distance of $53 \mathrm{~cm}$ from each other, equivalent to $1.35 \lambda$, assuring good de-correlation of the received signals. From the power delay profiles calculated for each branch, values of mean delay and RMS delay spread lead to conclude that the channel behavior was classified as suburban/ less dense urban, since the RMS delay spread varied from some nanoseconds to few microseconds with the major part of the values below 0.4 microseconds. The channel showed to be Gaussian and the improvement of the joint capacity has varied from 1 to $123 \mathrm{Mbps}$, totaling values from 4.72 Mbps to $260 \mathrm{Mbps}$ for the SIMO 1x2 system, therefore, showing that a simple diversity with two reception branches has reached 1.42 times the capacity of an isolated antenna.

\section{ACKNOWLEDGMENT}

Authors thank CAPES (Coordenação de Aperfeiçoamento de Pessoal de Nível Superior) by the scholarship provided and the RFS Company (Radio Frequency Systems), which donated the transmitter antenna. 


\section{REFERENCES}

[1] D. W. Matolak, K. A. Remley, C. Holloway, and C. Gentile, "Outdoor-to-Indoor Channel Dispersion and Power-Delay Profile Models for the 700-MHz and 4.9-GHz Bands," IEEE Antennas and Wireless Propag. Letters, vol. 15, 2016.

[2] C. Gentile et. al, "Modeling Urban Peer-to-Peer Channel Characteristics for the $700 \mathrm{MHz}$ and $4.9 \mathrm{GHz}$ Public Safety Bands," in IEEE International Conference on Communications, June 2012, Ottawa, Canada, pp.4557-4562.

[3] M. B. Moura, D. C. Vidal, C. Schueler, L. J. Matos and T. N. Ferreira, "Outdoor-to-Indoor Power Prediction for 768 $\mathrm{MHz}$ Wireless Mobile Transmission using Multilayer Perceptron," in IEEE World Congress on Computational Intelligence, 2018, Rio de Janeiro. 2018 Proceedings - International Joint Conference on Neural Networks (IJCNN), 2018. v. 1. pp. 1258-1264.

[4] D. Y. N. Cueto, L. A. R. Silva Mello, and C. V. R. Ron, "Comparison of Coverage and Capacity of LTE-Advanced Networks at $700 \mathrm{MHz}$ and $2.6 \mathrm{GHz}$," in International Microwave and Optical Conference - IMOC 2013, RJ, Brazil, pp. $1-5,2013$

[5] H. Ali, A. Doucet, and Y. Hua, "Blind SOS subspace channel estimation and equalization techniques exploiting spatial diversity in OFDM systems," Digital Signal Processing, Springer, vol. 14, no. 2, pp. 171-202, March 2004.

[6] K. Yagishita, Y. Kakishima, and M. Sawahashi,, "Effects of antenna receiver diversity with Faster-than-Nyquist signaling using OFDM/OQAM in multipath fading channel," in International Symposium on Wireless Personal Multimedia Communications (WPMC), 2014, pp. 351-355, Sep. 2014.

[7] G. B. Kim, J. Lee, Jac, and S. J. Hong, "Analysis of Macro-Diversity in LTE-Advanced," KSII Trans. on Internet and Information Systems, vol.5, no. 9, pp.1596-1612, Sep. 2011.

[8] J. D. Parsons, The Mobile Radio Propagation Channel, $2^{\text {nd }}$ edition, New York, John Wiley \& Sons, 2000.

[9] U. R. Villanueva, G. L. Siqueira, L. J. Matos, P. V. G. Castellanos, and L. H. G. Furtado, "Experimental Evaluation of the Mobile Radio Channel Capacity in the $2.48 \mathrm{GHz}$ Band," Journal of Microwaves, Optoelectronics and Electromagnetic Applications, v. 16, p. 471-480, 2017.

[10] C. D. Tse and P.Viswanath, Fundamentals of Wireless Communication USA, Cambridge University Press, 2005.

[11] J. Benesty et al., Noise Reduction in Speech Processing, Springer-Verlag, Berlin Heidlberg, 2009.

[12] T. S. Rappaport, Wireless Communications - Principles and Practice, $2^{\text {nd }}$. edition, Prentice Hall, 2002.

[13] C. Rauscher, Fundamental of Spectrum Analysis, Rohde \& Schwarz, Germany, 2001. 